\title{
Wild by Design: Workshop on Designing Ubiquitous Health Monitoring Technologies for Challenging Environments
}

\author{
Diogo Branco \\ djbranco@fc.ul.pt \\ LASIGE, Faculdade de Ciências da \\ Universidade de Lisboa \\ Portugal \\ Afsaneh Doryab \\ ad4ks@virginia.edu \\ University of Virginia \\ USA
}

\author{
Roisin McNaney \\ roisin.mcnaney@monash.edu \\ Monash University \\ Australia
}

\author{
Patrick Carrington \\ pcarring@andrew.cmu.edu \\ Carnegie Mellon University \\ USA
}

\author{
Hristijan Gjoreski \\ hristijang@feit.ukim.edu.mk \\ Ss. Cyril and Methodius University \\ North Macedonia
}

\author{
Kyle Montague \\ kyle.montague@northumbria.ac.uk \\ Northumbria University
}

UK

\section{Julio Vega \\ vegaju@upmc.edu \\ University of Pittsburgh}

\author{
Silvia Del Din \\ silvia.del-din@newcastle.ac.uk \\ Newcastle University \\ UK
}

Tiago Guerreiro

tjvg@di.fc.ul.pt

LASIGE, Faculdade de Ciências da

Universidade de Lisboa

Portugal

André Rodrigues

afrodrigues@fc.ul.pt

LASIGE, Faculdade de Ciências da

Universidade de Lisboa

Portugal

USA

\begin{abstract}
Recent years have shown an emergence of ubiquitous technologies that aim to monitor a person's health in their day to day. However, albeit focused at a real world setting and technically able, most research is still limited in its real-world coverage, suitability, and adoption. In this workshop, we will focus on the challenges of real world health monitoring deployments to produce forward-looking insights that can shape the way researchers and practitioners think about health monitoring, in platforms and systems that account for the complex environments where they are bound to be used.
\end{abstract}

\section{CCS CONCEPTS}

- Human-centered computing $\rightarrow$ Ubiquitous and mobile computing.

\section{KEYWORDS}

ubiquitous technologies, health, in-the-wild, longitudinal, challenges

\section{Unpublished working draft. Not for distribution.}

2021-04-01 10:00. Page 1 of 1-4.
ACM Reference Format:

Diogo Branco, Patrick Carrington, Silvia Del Din, Afsaneh Doryab, Hristijan Gjoreski, Tiago Guerreiro, Roisin McNaney, Kyle Montague, André Rodrigues, and Julio Vega. 2018. Wild by Design: Workshop on Designing Ubiquitous Health Monitoring Technologies for Challenging Environments. In Woodstock '18: ACM Symposium on Neural Gaze Detection, June 03-05, 2018, Woodstock, NY. ACM, New York, NY, USA, 4 pages. https: //doi.org/10.1145/1122445.1122456

\section{INTRODUCTION}

There is a growing focus on monitoring health in uncontrolled (aka in-the-wild) settings. This is partially due to the limitations of occasional formal assessments, and to the increasing availability of off-the-shelf sensing devices and applications. This change of paradigm promises fine-grained monitoring of chronic and neurodegenerative diseases, early prediction of health conditions, accessible self-management, and the enablement of more effective personalised interventions. Recent research shows us the potential of these ubiquitous technologies: with body-worn accelerometer sensors, it is possible to monitor disease fluctuations in free-living environments [1, 5, 16]; predict hospital readmission [10]; monitor depression via social media posts [4]; detect continuous exposure to stress with wrist-worn devices[6]; use smartphones to passively monitor diseases $[8,15]$ or perform rapid tests in low-resource settings [14]; among many others.

Despite this potential, there are still limited examples of ubiquitous health technologies used in practice for long periods of time 
[12]. The majority of ubiquitous health technologies, although targeted at uncontrolled in-the-wild settings, are still mostly tested in the laboratory. Activity trackers are one successful example of a technology that became mainstream. However, it also faces problems with abandonment and adoption [3,9] and misalignment with user requirements and goals [2]. Although the need for longitudinal in-the-wild studies has been established [7,13], the challenges of engaging and sustaining participation has only been surfaced [11]. Awareness and knowledge of this and other challenges for in-the-wild health monitoring is still very limited.

In this workshop, we will discuss the challenges of "real life" health monitoring technology deployments, and shift the dialogue from a focus on technical feasibility to real-world feasibility and relevance, namely regarding inclusion, end-user acceptance, robustness, and sustainability of solutions. Particularly, we will use case studies and examples of deployments and tools as a basis for discussion of challenging environments, and itemize barriers, requirements, and solutions for the future of ubiquitous health monitoring.

\section{WORKSHOP GOALS}

One of the main goals of the workshop is to bridge cross-disciplinary relationships between researchers and practitioners interested in the design, development, and deployment of ubiquitous technologies for health monitoring. What major challenges arise in in-thewild deployments of ubiquitous technology? How can the data streams be interpretable and actionable for end-users and health professionals? What are the best practices for engaging and sustainable participation in longitudinal deployments?

To answer these example questions and others, we hope to bring together the ubiquitous computing, sensor development, machine learning, digital health, clinical, HCI and accessibility communities. Through this workshop, participants will be able to share real-life deployment experiences, tools, challenges, and discuss designs and technology goals for future research. Some of the specific areas workshop participants may have experience with include:

- Understanding clinical, contextual, and individual health monitoring needs and constraints;

- Understanding cultural, geographical, social, and economic aspects of health technologies;

- Understanding chronic conditions, requirements, and limitations;

- Designing and developing longitudinal health monitoring studies;

- Development of tools and platforms for ubiquitous health monitoring;

- Development of tools and methods to analyse in-the-wild longitudinal data;

- Validation of metrics extracted from sensor data as human behaviour proxies or clinical end-points.

- Understanding the ethical, privacy, and sharing aspects of everyday technologies.

Additional workshop goals include:

- Highlight future challenges for this community to guide future research;

- Create awareness for the idiosyncrasies of the real world;
- Identify requirements and conflicts in emergent and unsung scenarios and contexts;

- Identify guidelines for researchers to reduce the gap between laboratorial and in-the-wild research;

- Build a cross-disciplinary community on ubiquitous health monitoring technologies;

- Share best practices.

\section{WORKSHOP TOPICS}

The workshop organizers' background is diverse and includes experience in developing ubiquitous tools for health monitoring, free-living disease assessment and monitoring, activity recognition, inclusive activity tracking, human-computer interaction and accessibility.

Topics for the workshop include:

- Longitudinal deployments and studies

- Contextual challenges of in-the-wild deployments

- Compliance with longitudinal studies

- Tools for passive and active monitoring

- Socio-economic and cultural factors of influence

- Engaging and sustaining participants in longitudinal research and practice

- Disruption and decay to digital infrastructures

- Sharing and privacy of longitudinal data

- Everyday Ethical Dilemmas

- End-user interfaces and benefits of sensing technologies

- Unintentional learning and consequences of ubiquitous technology deployments

- Dynamic diversity and adaptation

- Visualization of longitudinal data (e.g., clinicians and self)

- Conflicts between adoption and study integrity

\section{WORKSHOP SUBMISSION}

The workshop will invite submissions of case studies, position papers or provocations, and demonstrations that describe ubiquitous health monitoring deployments, tools, and/or challenging environments. Submissions should use the ACM sigconf template (https://www.acm.org/publications/proceedings-template) and have a maximum of 4 pages.

\section{WORKSHOP FORMAT}

The workshop will be open to everyone who wishes to participate, even if they haven't submitted a contribution.

Activities of the workshop will start before Ubicomp. We will ask authors to create 5 minute presentations of their submissions, and make them available in a workshop Discord channel (together with the paper and possible links - e.g., in the case of platforms and tools). In there, we will launch discussions on each submission, enabling Q\&A long before the workshop. Based on interest, we will create short cross-disciplinary working groups, that will carry on discussions, identify challenges, and ideas and best practices.

During the day of the workshop, we will dedicate time to discuss individual contributions, but focus most time on summary of group discussions. These groups will focus on creating design fictions of challenging environments informed by the submissions. We are planning the usage of Miro boards as a way to support and 
document the group activities. After each discussion period, groups will report to everyone. A live document with challenges and best practices will serve as a report and shared output from this town hall activity.

After the workshop, we plan to continue editing the live document, and make it available openly as a white paper. Submissions to the workshop, that are in scope, will be invited to submit extended versions to special issues of MDPI Sensors (on Passive Sensing for Health) and ACM TACCESS (on Inclusive Health Monitoring). Also, we plan to engage participants in the organization of the next Wild by Design edition, at Ubicomp.

\section{REFERENCES}

[1] David Andre and Donna L Wolf. 2007. Recent advances in free-living physical activity monitoring: a review. Fournal of diabetes science and technology 1, 5 (2007), 760-767.

[2] Patrick Carrington, Kevin Chang, Helena Mentis, and Amy Hurst. 2015. " But, I don't take steps" Examining the Inaccessibility of Fitness Trackers for Wheelchair Athletes. In Proceedings of the 17th international acm sigaccess conference on computers \& accessibility. 193-201.

[3] James Clawson, Jessica A. Pater, Andrew D. Miller, Elizabeth D. Mynatt, and Lena Mamykina. 2015. No Longer Wearing: Investigating the Abandonment of Personal Health-Tracking Technologies on Craigslist. In Proceedings of the 2015 ACM International foint Conference on Pervasive and Ubiquitous Computing (Osaka, Japan) (UbiComp '15). Association for Computing Machinery, New York, NY, USA, 647-658. https://doi.org/10.1145/2750858.2807554

[4] Munmun De Choudhury, Michael Gamon, Scott Counts, and Eric Horvitz. 2013 Predicting depression via social media. In Proceedings of the International AAAI Conference on Web and Social Media, Vol. 7.

[5] Silvia Del Din, Alan Godfrey, Claudia Mazzà, Sue Lord, and Lynn Rochester. 2016 Free-living monitoring of Parkinson's disease: Lessons from the field. Movement Disorders 31, 9 (2016), 1293-1313.

[6] Martin Gjoreski, Hristijan Gjoreski, Mitja Luštrek, and Matjaž Gams. 2016 Continuous Stress Detection Using a Wrist Device: In Laboratory and Real Life. In Proceedings of the 2016 ACM International foint Conference on Pervasive and Ubiquitous Computing: Adjunct (Heidelberg, Germany) (UbiComp '16). Association for Computing Machinery, New York, NY, USA, 1185-1193. https://doi.org/10.1145/2968219.2968306

[7] Ilkka Korhonen, Juha Parkka, and Mark Van Gils. 2003. Health monitoring in the home of the future. IEEE Engineering in medicine and biology magazine 22, 3 (2003), 66-73.

[8] Elina Kuosmanen, Florian Wolling, Julio Vega, Valerii Kan, Yuuki Nishiyama, Simon Harper, Kristof Van Laerhoven, Simo Hosio, and Denzil Ferreira. 2020 Smartphone-Based Monitoring of Parkinson Disease: Quasi-Experimental Study to Quantify Hand Tremor Severity and Medication Effectiveness. FMIR mHealth and uHealth 8, 11 (2020), e21543.

[9] Amanda Lazar, Christian Koehler, Theresa Jean Tanenbaum, and David H. Nguyen. 2015. Why We Use and Abandon Smart Devices. In Proceedings of the 2015 ACM International foint Conference on Pervasive and Ubiquitous Computing (Osaka, Japan) (UbiComp '15). Association for Computing Machinery, New York, NY, USA, 635-646. https://doi.org/10.1145/2750858.2804288

[10] Carissa A Low, Dana H Bovbjerg, Steven Ahrendt, M Haroon Choudry, Matthew Holtzman, Heather L Jones, James F Pingpank Jr, Lekshmi Ramalingam, Herbert J Zeh III, Amer H Zureikat, et al. 2018. Fitbit step counts during inpatient recovery from cancer surgery as a predictor of readmission. Annals of Behavioral Medicine 52,1 (2018), 88-92.

[11] Gonzalo J Martinez, Stephen M Mattingly, Shayan Mirjafari, Subigya K Nepal, Andrew T Campbell, Anind K Dey, and Aaron D Striegel. 2020. On the Quality of Real-world Wearable Data in a Longitudinal Study of Information Workers. In 2020 IEEE International Conference on Pervasive Computing and Communications Workshops (PerCom Workshops). IEEE, 1-6.

[12] J. Meyer, E. Beck, M. Wasmann, and S. Boll. 2017. Making Sense in the Long Run: Long-Term Health Monitoring in Real Lives. In 2017 IEEE International Conference on Healthcare Informatics (ICHI). 285-294. https://doi.org/10.1109/ICHI.2017.11

[13] Kyle Montague, Hugo Nicolau, and Vicki L Hanson. 2014. Motor-impaired touchscreen interactions in the wild. In Proceedings of the 16th international ACM SIGACCESS conference on Computers \& accessibility. 123-130.

[14] Chunjong Park, Alex Mariakakis, Jane Yang, Diego Lassala, Yasamba Djiguiba, Youssouf Keita, Hawa Diarra, Beatrice Wasunna, Fatou Fall, Marème Soda Gaye, Bara Ndiaye, Ari Johnson, Isaac Holeman, and Shwetak Patel. 2020. Supporting Smartphone-Based Image Capture of Rapid Diagnostic Tests in Low-Resource Settings. In Proceedings of the 2020 International Conference on Information and Communication Technologies and Development (Guayaquil, Ecuador) (ICTD2020).

2021-04-01 10:00. Page 3 of 1-4
Association for Computing Machinery, New York, NY, USA, Article 2, 11 pages. https://doi.org/10.1145/3392561.3394630

[15] Yonatan Vaizman, Katherine Ellis, and Gert Lanckriet. 2017. Recognizing detailed human context in the wild from smartphones and smartwatches. IEEE pervasive computing 16, 4 (2017), 62-74.

[16] Ruben PA van Eijk, Jaap NE Bakers, Tommy M Bunte, Arianne J de Fockert, Marinus JC Eijkemans, and Leonard H van den Berg. 2019. Accelerometry for remote monitoring of physical activity in amyotrophic lateral sclerosis: a longitudinal cohort study. fournal of neurology 266, 10 (2019), 2387-2395.

\section{A CALL FOR PAPERS}

The main goal of this workshop is to bridge cross-disciplinary relationships between researchers and practitioners interested in the design, development, and deployment of ubiquitous technologies for health monitoring. Which major challenges arise in in-the-wild deployments of ubiquitous technology? How can the streams of data be manageable for end-users and health professionals? What are the best practices for engaging and sustainable participation in longitudinal deployments? Which are socio-economic, cultural and individual factors of influence?

To answer these example questions and others, we hope to bring together the ubiquitous computing, sensor development, machine learning, digital health, clinical, $\mathrm{HCI}$ and accessibility communities. Through this workshop, participants will be able to share real-life deployment experiences, tools, challenges, and discuss designs and technology goals for future research.

\section{A.1 Topics}

Topics for the workshop include:

- Longitudinal deployments and studies

- Contextual challenges of in-the-wild deployments

- Compliance with longitudinal studies

- Tools for passive and active monitoring

- Socio-economic and cultural factors of influence

- Engaging and sustaining participants in longitudinal research and practice

- Disruption and decay to digital infrastructures

- Sharing and privacy of longitudinal data

- Everyday Ethical Dilemmas

- End-user interfaces and benefits of sensing technologies

- Unintentional learning and consequences of ubiquitous technology deployments

- Dynamic diversity and adaptation

- Visualization of longitudinal data (e.g., clinicians and self)

- Conflicts between adoption and study integrity

\section{A.2 Submission}

We invite submissions of case studies, position papers or provocations, and demonstrations that describe ubiquitous health monitoring deployments, tools, and/or challenging environments. Submissions should use the ACM sigconf template ${ }^{1}$ and have a maximum of 4 pages.

\section{A.3 Important Dates}

- Submission deadline: June 15th, 2021

- Notification: July 15 th, 2021

$\overline{{ }^{1} \text { https://www.acm.org/publications/proceedings-template }}$ 
- Workshop: September 25th or 26th, 2021

\section{A.4 Organizers}

- Diogo Branco, LASIGE, Universidade de Lisboa, Portugal - Patrick Carrington, Carnegie Mellon University, USA

- Silvia Del Din, Newcastle University, UK

- Afsaneh Doryab, University of Virginia, USA
- Hristijan Gjoreski, Ss. Cyril and Methodius University, North Macedonia

- Tiago Guerreiro, LASIGE, Universidade de Lisboa

- Roisin McManey, Monash University, Australia

- Kyle Montague, Northumbria University, UK

- André Rodrigues, LASIGE, Universidade de Lisboa, Portugal

- Julio Vega, University of Pittsburgh, USA
407 408 